\title{
Interactive comment on "Brown carbon's emission factors and optical characteristics in household biomass burning: Developing a novel algorithm for estimating the contribution of brown carbon" by Jianzhong Sun et al.
}

Anonymous Referee \#2

Received and published: 25 August 2020

This paper presents emission factors for brown carbon and black carbon from 11 different biomass fuels in a commonly used cookstove. Most of the paper is focused on the development of an algorithm to convert $\mathrm{AAE}$ into the mass ratio of $\mathrm{BrC}$ to $\mathrm{BC}$ and solar absorption fraction attributed to $\mathrm{BrC}$. The paper makes the important point that $\mathrm{BrC}$ absorption needs to be included in assessments of the climate impacts from biomass burning. Given the data presented here (one stove and several biomass fuels), the universality of the algorithm for multiple emission sources is overstated. Also, there is limited comparison of the algorithm with other methods, so it is not clear if it is 
an improvement over other approaches of estimating the impact of $\mathrm{BrC}$ on climate. I recommend major revisions to address the following comments:

General Comments:

1. The quantification of $\mathrm{BrC}$ as a mass emission is a relatively uncommon approach due to the complexity of $\mathrm{BrC}$ (i.e. many different chromophores with differing mass absorption efficiencies that are source dependent). A fuller description of the proxies used in this current study and how they compare to other black and brown carbon sources should be outlined (e.g. absorption efficiencies, AAE, primary particle size, etc.) and any shortcomings of using these proxies should be noted. How does this method of estimating $\mathrm{BrC}$ mass emissions compare with other approaches used in the literature that were cited for comparison of $\mathrm{BrC}$ emission factors (e.g. Aurell and Gullett 2013 and Schmidl et al. 2008)?

2. More description of the test protocol is needed, e.g. cold start? size of fuel? How was it determined that the test method was relevant for real-world stove emissions?

3. Overall, there needs to be an analysis of the uncertainty or the error and potential impacts of the assumptions in the algorithm? What is the impact of assuming $A A E=1$ for $\mathrm{BC}$ ? How might lensing impact this analysis? What is the impact of measurement limit of detection?

4. How does the novel algorithm presented here compare with other approaches to quantifying the fractional contribution of $\mathrm{BrC}$ and $\mathrm{BC}$ to absorption? Is there a benefit to calculating a $\mathrm{BrC}$ mass emission factor over other approaches based on AAE? A few studies that may pertain might be Corbin et al. 2018 (https://doi.org/10.1029/2017JD027818), Tian et al. 2019 (https://doi.org/10.1029/2018JD029352), or Zhang et al. 2018 (https://aaqr.org/articles/aaqr-17-12-ac3-0566.pdf) among many others.

5. There is no validation that this algorithm works for sources other than the cookstove 
samples measured in this study and in Sun et al. 2017. Unless the authors can include some additional data points from some other sources in their algorithm development the statements made throughout the paper about the wide applicability of the algorithm for 'any combustion sources' are unsupported and should be removed.

6. The manuscript needs to be edited for language, see minor comments for specific examples. But generally, if you are writing 'in other words' it means your first explanation should be simplified and stated only once.

Specific Comments:

Line 30-31: The sentence needs to be rewritten since the two clauses of this sentence are saying the same thing, $\mathrm{BrC}$ absorbs more at shorter wavelengths.

Line 46: Why are the units in $\mathrm{mg} / \mathrm{m} 2$ ? Most species in the atmosphere are reported in terms of concentration. Is this a typo?

Line 71: Need to be clearer about what characteristics are being referred to here. There are many references published on emissions which measure chemical composition, size distribution, and some even quantify optical properties. Most do not report a $\mathrm{BrC}$ emission factor because there is no standard for quantifying $\mathrm{BrC}$ mass.

Line 114: These 'soft materials' are usually referred to as kindling and is commonly used when igniting wood and the emissions from a kindling ignition should be included in this analysis since they are representative of real-world use.

Line 120: What is meant by 'envisaged emission intensity'? How was this determined? Is this just the concentration in the sampling duct?

Line 172: Please cite a reference and quantify how much lower the burning temperature or heat release is for herbaceous fuels to support this speculation.

Lines 175 - 181: Were no other measurements made during the tests (e.g. CO, CO2, $\mathrm{PM}, \mathrm{EC}, \mathrm{OC})$ ? These other measurements would greatly support some of the specu- 
lation in this section. I am not sure the speculation is justified without measurements from actual study here.

Lines 205: This paragraph needs to be revised for language usage.

Line 208: What is meant by 'the significant potential of $\mathrm{BrC}$ emissions than $\mathrm{BC}$ emissions'? Does this mean larger emissions? Larger mass fractions? Larger $\mathrm{BrC} / \mathrm{BC}$ ratios? Larger impact? Be specific about what quantity is of $\mathrm{BrC}$ emissions is significant and by what amount.

Line 212: Please provide the average absorption efficiencies of $\mathrm{BrC}$ and $\mathrm{BC}$ that are being referenced for this statement.

Line 255-57: Why are funeral pyres used as an emissions comparison? It seems like an odd source to include and to leave out any mention of open burning (e.g. ag residues, forest fires) or coal for cookstoves. Is coal included in the 'biomass fuels' in mentioned in line 255?

Line 280: What was the source of the uncertainties in the Lack and Langridge analysis? Do they apply in this study?

Line 328-331: How does this compare to the direct radiative forcing attributed to $\mathrm{BrC}$ referred to in the introduction?

Figure 1: Please include error bars to show the uncertainty in the measurement. Presumabley repeat measurements were made because there are standard deviations (standard error?) provided in Table 1.

Table 1: Please include all the quantities measured and calculated for each sample (e.g. AAE, RBrC/BC, $\mathrm{fBrC}, \mathrm{FBrC}$ ) along with propagated uncertainties.

Figure 4: What is the impact of limit of detection on this plot? The data $>750 \mathrm{~nm}$ is very noisy, and I wonder if that is not due to limitations of the measurement? If this data is below the limit of detection it should not be used in the calculation of $\mathrm{fBrC}$. 
Figure 5 and Line 303: Why only use the mean (median?) fBrC from these current study and Sun et al. 2017? Although the regression is strongly correlated here, the scatter in the data is covered up by using the mean value instead of every measured data point.

SI:

Tables S3 Part I: Extracts are dominated by ambient aerosols, what about source? E.g. fossil fuel combustion, woodstoves, open burning? (Just a few examples are: Xie et al. 2017 https://doi.org/10.1038/s41598-017-06981-8 for open burning and gasoline exhaust; Xie et al. 2018 https://doi.org/10.1016/j.envpol.2018.04.085 for cookstoves using wood, kerosene and charcoal; Corbin et al. 2018 https://doi.org/10.1029/2017JD027818 for marine diesel engines). Since this paper is focused on emissions it would be good to have a more exhaust list of emissions AAE measurements. Calculations: should 'coal' here be 'biomass fuel'?

Figures S2-S4: Appear to be identical to those in Sun et al. 2017, should the reference be noted in the caption?

Figure S4: Hard to follow the text here, would be easier to understand in equation form or even a diagram.

Interactive comment on Atmos. Chem. Phys. Discuss., https://doi.org/10.5194/acp-2020-548, 2020. 